\title{
Continuous-flow catalytic asymmetric hydrogenations: Reaction optimization using FTIR inline analysis
}

\author{
Magnus Rueping ${ }^{*}$, Teerawut Bootwicha and Erli Sugiono
}

\section{Full Research Paper}

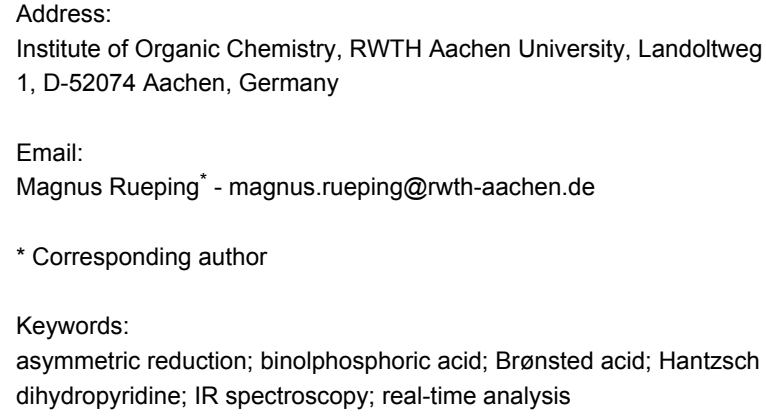

\section{Open Access}

\author{
Beilstein J. Org. Chem. 2012, 8, 300-307. \\ doi:10.3762/bjoc. 8.32 \\ Received: 03 January 2012 \\ Accepted: 13 February 2012 \\ Published: 23 February 2012 \\ This article is part of the Thematic Series "Chemistry in flow systems II". \\ Guest Editor: A. Kirschning \\ (C) 2012 Rueping et al; licensee Beilstein-Institut. \\ License and terms: see end of document.
}

\begin{abstract}
The asymmetric organocatalytic hydrogenation of benzoxazines, quinolines, quinoxalines and $3 H$-indoles in continuous-flow microreactors has been developed. Reaction monitoring was achieved by using an inline ReactIR flow cell, which allows fast and convenient optimization of reaction parameters. The reductions proceeded well, and the desired products were isolated in high yields and with excellent enantioselectivities.
\end{abstract}

\section{Introduction}

In recent years, a growing interest in microreactor technology has been seen in the scientific community and the development of microfabricated reaction systems is actively pursued. Microreactor technology offers numerous advantages, including precise control of reaction variables, enhanced mixing quality, improved operational safety, reduced reagent consumption and ready scale-up of chemical processes. Due to the high surfacearea-to-volume ratios of microstructured reactors, a high thermal rate and high portability of substrates can be achieved, which leads to improved product formation [1-42]. Furthermore, by incorporating inline analytical devices the progress of reactions can be monitored and analyzed in real time, allowing fast reaction screening and optimization [43-55].
Continuous flow microreactors have been applied to a number of standard transformations in organic synthesis [56-80]; however, examples regarding asymmetric reactions as well as organocatalytic reactions are scarce [81-96]. Herein, we present the first example of a continuous-flow organocatalytic asymmetric transfer hydrogenation performed in a microreactor. In this work a ReactIR flow cell was coupled with the microreactor and applied as an inline monitoring device for optimizing the reactions.

\section{Results and Discussion}

The continuous-flow microreactor system for the experiment was set up according to Scheme 1. The flow device was set up 
either with a single reactor, or with multiple reactors when a prolonged residence time was needed. The reagents were introduced separately, by using a syringe pump, through two inlets connected to $\mathrm{Y}$-shaped connectors. The internal reaction temperature was monitored with an internal thermal sensor. The ReactIR 45m microflow cell equipped with a DiComp ATR (diamond-composite attenuated total reflection) probe was attached to the microreactor at the end of the reaction stream and was used as an inline analytical tool to determine the optimum reaction conditions. The IR spectra were recorded at predefined intervals and the raw data were analysed with iC-IR analysis software.

The first reaction examined the asymmetric organocatalytic transfer hydrogenation [97-101] of benzoxazine 3a in the presence of Hantzsch dihydropyridine $\mathbf{2 a}$ as hydrogen source and a catalytic amount of chiral Brønsted acid 1a (Scheme 2) [102].

Initial experiments were carried out at $0.1 \mathrm{~mL} \mathrm{~min}^{-1}$ flow rate in a commercial glass microreactor, which was attached to the ReactIR flow cell for in situ reaction monitoring. In order to

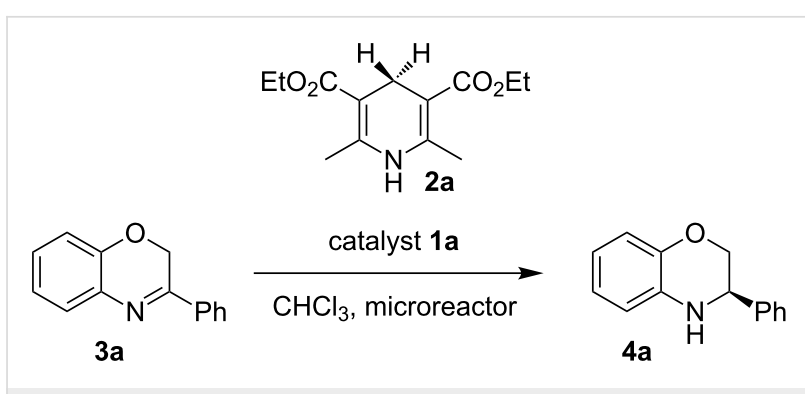

Scheme 2: Asymmetric hydrogenation of benzoxazines.

control the reaction and to determine the use of educts and formation of product, reference spectra of the starting materials, solvents and reagents were recorded. Figure $1 \mathrm{~b}$ and Figure 1c show real time IR spectra of the reaction mixtures after the subtraction of solvent in the spectral region of 1440 and $1530 \mathrm{~cm}^{-1}$. For direct inline analysis the signals at $\tilde{v}=$ $1479 \mathrm{~cm}^{-1}$ and $\tilde{v}=1495 \mathrm{~cm}^{-1}$ were ideal as they could easily be assigned to benzoxazine $\mathbf{3 a}$ and dihydrobenzoxazine $\mathbf{4 a}$. Thus, in continuous flow the substrate consumption and product formation could readily be determined.

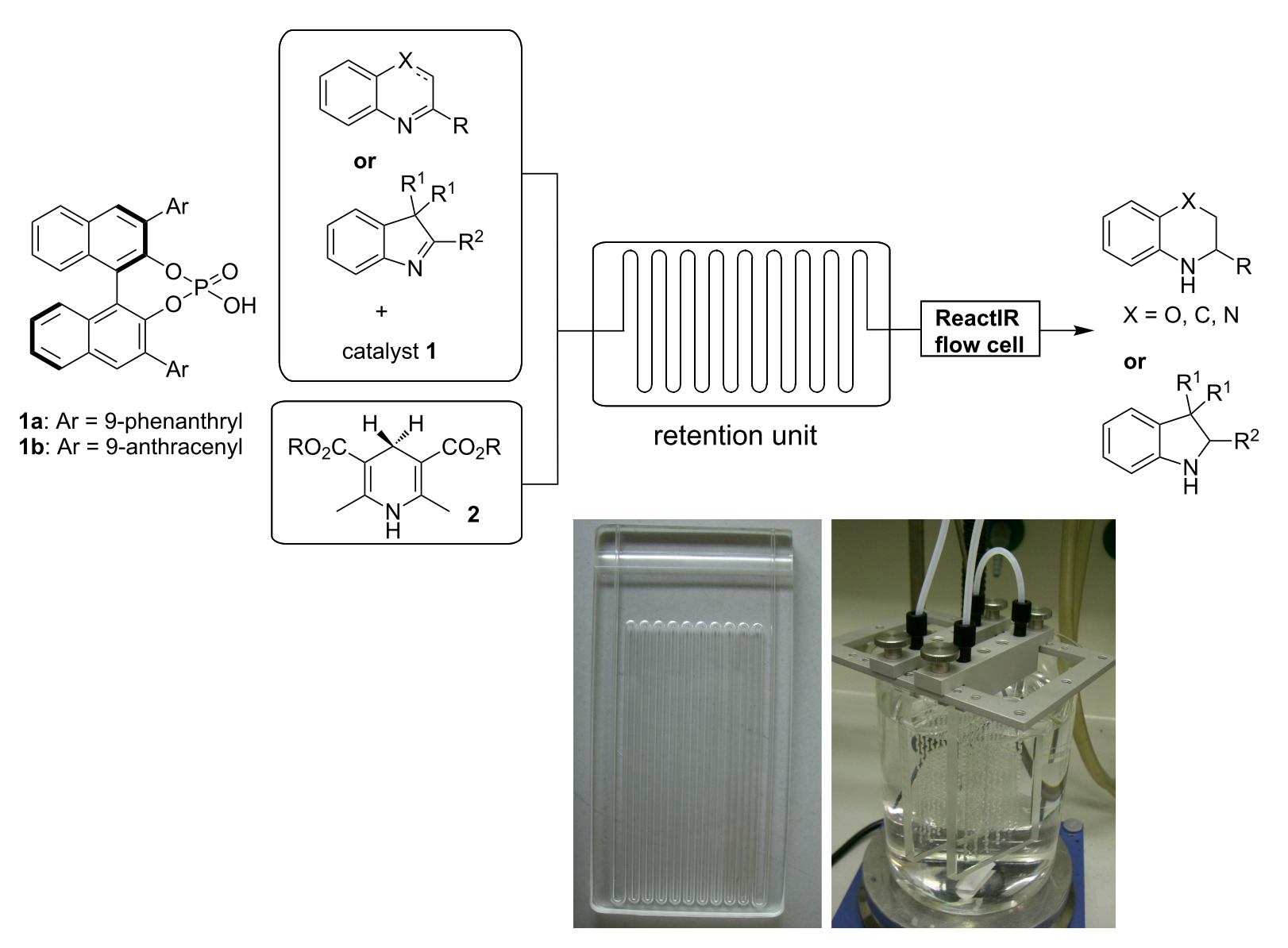




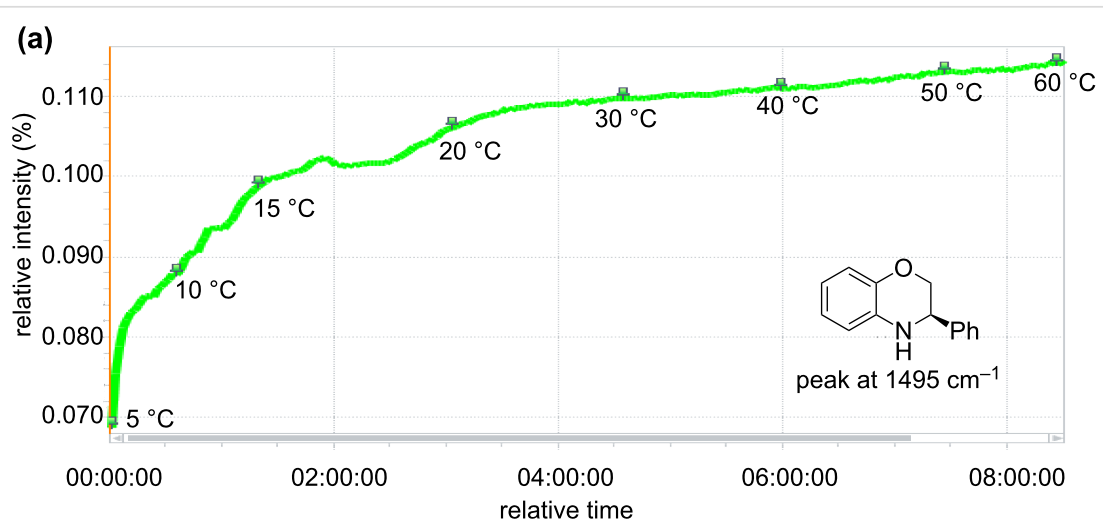

(b)

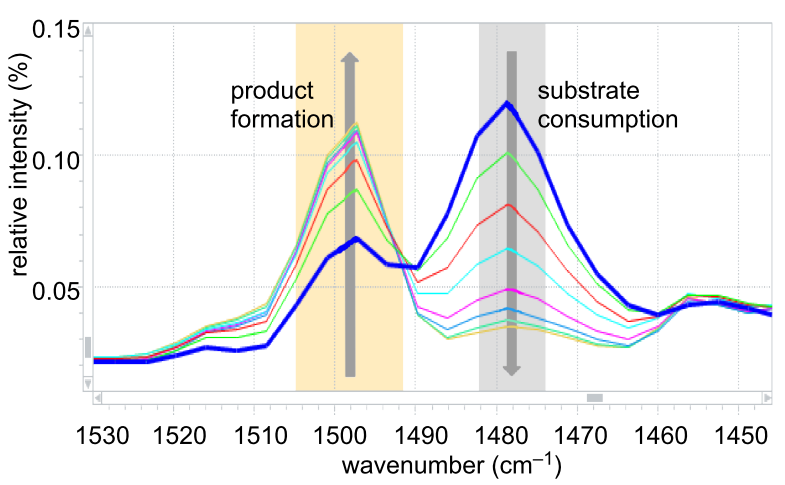

(c)

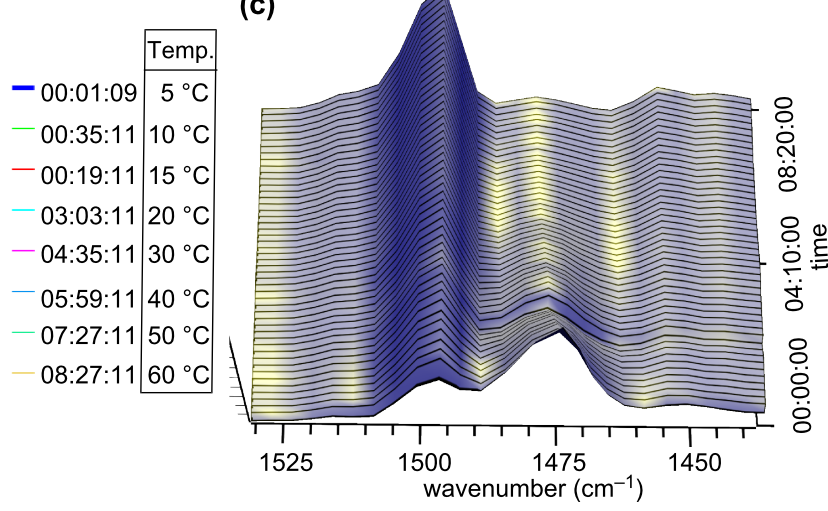

Figure 1: In situ ReactIR monitoring: (a) Trend curve of product formation at different temperatures. (b) Reaction spectra showing the consumption of the substrate and the formation of product at different temperatures. (c) Three-dimensional time-resolved spectral data.

In order to find the optimal temperature for the asymmetric continuous-flow reduction, a temperature profile was recorded. The reaction temperature was initially $5{ }^{\circ} \mathrm{C}$ and was increased to $60{ }^{\circ} \mathrm{C}$ over a period of $8 \mathrm{~h}$, while the conversion was monitored by inline IR-spectroscopy. Figure 1a shows the real-time plot of the peak intensity versus reaction time for the $1495 \mathrm{~cm}^{-1}$ absorption band at different temperatures. The trend-curve analysis by peak-height integration of this absorption band shows increased product formation with increasing temperature. By monitoring the signal change in this spectral region over the time of the reaction, the product formation $\left(\tilde{v}=1495 \mathrm{~cm}^{-1}\right)$ and substrate consumption $\left(\tilde{v}=1479 \mathrm{~cm}^{-1}\right)$ can be determined in real time. Analysis of the spectra provided us with an optimal temperature of $60{ }^{\circ} \mathrm{C}$ for this reaction. In general the IR-flowcell technology is a good tool for in situ monitoring and provides a fast read out of reaction progress as the intensity of substrate and product peaks can be directly related to the conversion. Thus, as exemplified above, applying the inline analysis to different reaction parameters provides a fast and convenient method for reaction optimization.

By using the optimized reaction temperature and flow rate of $0.1 \mathrm{~mL} \mathrm{~min}^{-1}$, further experiments were conducted to examine the influence of the residence time on the conversion (Table 1).
By performing the reaction with a residence time of $20 \mathrm{~min}$, the product was isolated in $50 \%$ yield. With residence times of $40 \mathrm{~min}$ and $60 \mathrm{~min}$, the product was isolated in $87 \%$ and $98 \%$ yields, respectively (Table 1).

Table 1: Optimization of the Brønsted acid catalyzed reduction of benzoxazines. $^{a}$

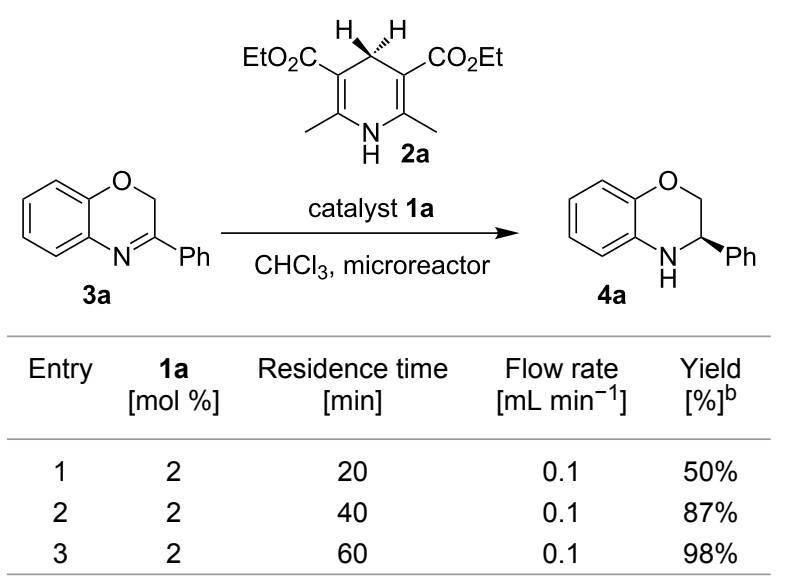

aReaction conditions: 3a, 2a (1.2 equiv), $\mathbf{1 a}$ in $\mathrm{CHCl}_{3}(0.05 \mathrm{M})$ at $60{ }^{\circ} \mathrm{C}$. ' Isolated yields after column chromatography. 
Having found the optimum reaction conditions, we next investigated the scope of the Brønsted acid catalyzed reduction of 3-aryl-substituted benzoxazines 3 (Table 2). In general, 3-aryl benzoxazines 3 bearing either electron-withdrawing or electrondonating groups can be reduced in a continuous fashion and the products 4 were isolated in good yields and with excellent enantioselectivities.

Encouraged by the results, we next studied the transfer hydrogenation of quinolines 5 [103-106]. The optimum reaction temperature was determined according to the experiment

Table 2: Scope of the Brønsted acid catalyzed reduction of benzoxazines. ${ }^{a}$
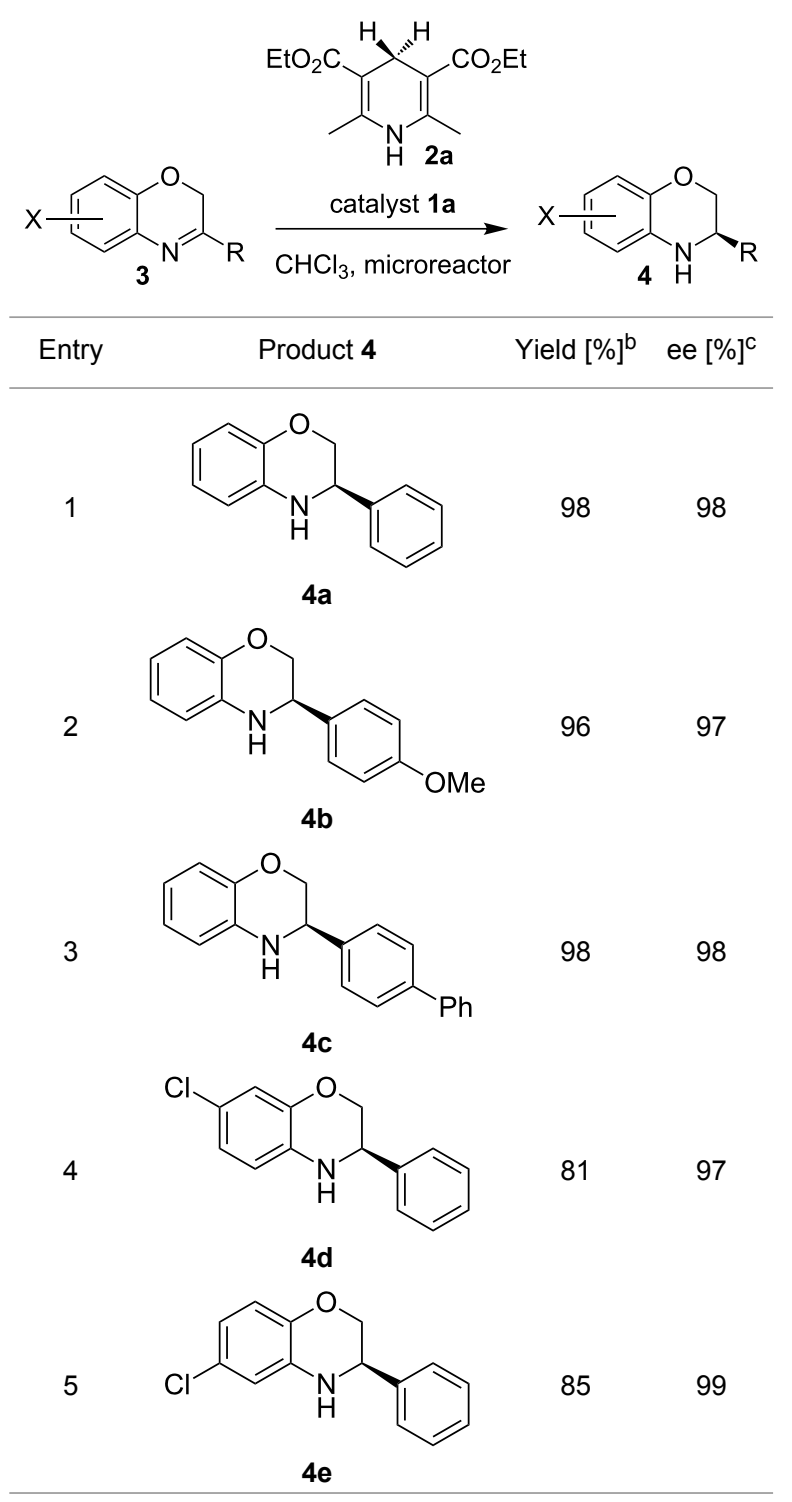

aReaction conditions: 3, 2a (1.2 equiv), 2 mol \% 1a in $\mathrm{CHCl}_{3}(0.05 \mathrm{M})$ at $60{ }^{\circ} \mathrm{C}$, flow rate $0.1 \mathrm{~mL} \mathrm{~min}^{-1}$, residence time $=60 \mathrm{~min}$. ${ }^{\mathrm{b}}$ Isolated yields after column chromatography. ${ }^{~}$ Determined by chiral HPLC analysis. described above. The effects of catalyst loading and residence time on the conversion and the enantioselectivity are summarized in Table 3. Performing the reaction at $60^{\circ} \mathrm{C}$ with $5 \mathrm{~mol} \%$ of Brønsted acid 1a and residence time of $20 \mathrm{~min}$ afforded the desired product in $88 \%$ yield and $94 \%$ enantioselectivity (Table 3, entry 1). When the catalyst loading was reduced from $5 \mathrm{~mol} \%$ to $2 \mathrm{~mol} \%$, a residence time of $40 \mathrm{~min}$ was found to be optimal to achieve comparable results (Table 3, entry 1 versus entry 2). A slight improvement of the conversion was observed by increasing the residence time to $60 \mathrm{~min}$ (Table 3 , entry 3 versus entry 2). The catalyst loading can be decreased to $0.5 \mathrm{~mol} \%$ without loss of reactivity and selectivity; the desired tetrahydroquinoline was isolated in $96 \%$ yield with $94 \%$ enantiomeric excess (Table 3, entry 5). A further decrease of catalyst loading to $0.1 \mathrm{~mol} \%$ resulted in a significant drop in chemical yield, affording the product in lower yield while enantioselectivity was maintained (Table 3 , entry 6 ).

Although continuous-flow reactions provide many advantages, in certain cases it can be beneficial to conduct reactions under classical batch conditions. Therefore, we decided to carry out a direct comparison. Transferring the reaction conditions from continuous-flow to the batch showed a noticeable drop in conversion and the product was isolated only in $67 \%$ yield (Table 3, entry 5 vs entry 7). This observation is general, and typically lower reactivities were obtained. This can be explained by the better heat transfer in the microreactors as compared to the glass flask typically used in our batch reactions.

Table 3: Optimization of the Brønsted acid catalyzed transfer hydrogenation of quinolines. $^{a}$

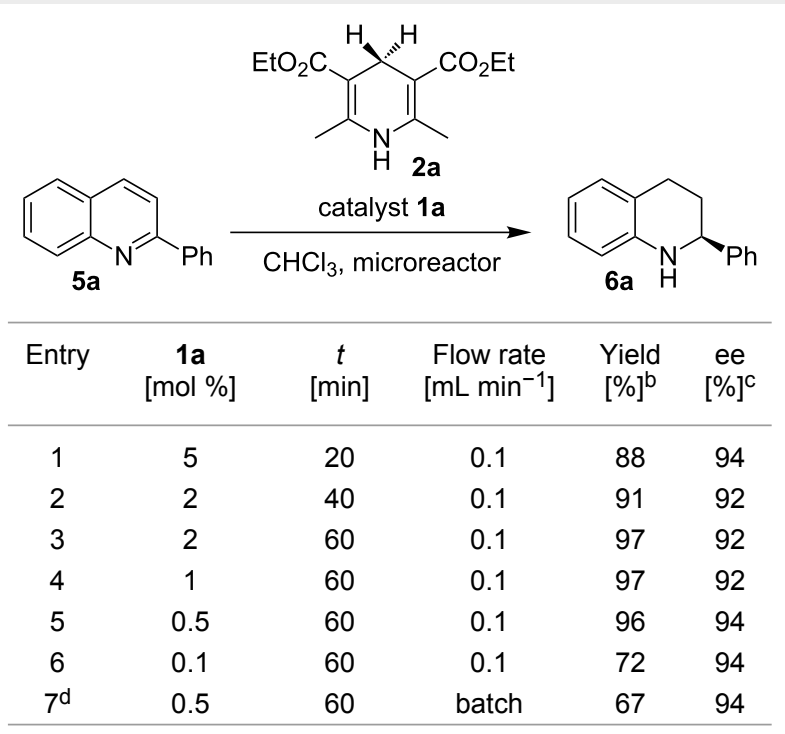

aReaction conditions: $\mathbf{5 a}, \mathbf{2 a}$ (2.4 equiv), $\mathbf{1 a}$ in $\mathrm{CHCl}_{3}(0,1 \mathrm{M})$ at $60^{\circ} \mathrm{C}$, flow rate $0.1 \mathrm{~mL} \mathrm{~min}^{-1}$. bIsolated yields after column chromatography. 'Determined by chiral HPLC analysis. ${ }^{\mathrm{C}}$ Performed under batch conditions. 
The scope and applicability of the method was then tested on various 2-substituted quinolines (Table 4). In general the asymmetric continuous-flow transfer hydrogenation of 2-substituted quinolines 5 proceeded well and afforded tetrahydroquinolines 6a-e with excellent yields and enantioselectivities (Table 4).

Having established a protocol for a general and highly enantioselective transfer hydrogenation of quinolines, we decided to extend its scope to the reduction of quinoxalines 7 (Table 5) [107]. The asymmetric reduction of quinoxalines is typically

Table 4: Scope of the Brønsted acid catalyzed transfer hydrogenation of quinolines. ${ }^{a}$

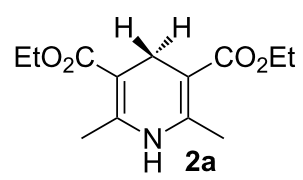<smiles>[R]c1ccc2ccccc2n1</smiles><smiles>CC(C)(C)C(C)(C)C(=O)OC(=O)Cl</smiles><smiles>[R]C1CCc2ccccc2N1</smiles>

\begin{tabular}{llll}
\hline Entry & Product 6 & $\begin{array}{l}\text { Yield } \\
{[\%]^{\mathrm{b}}}\end{array}$ & $\begin{array}{c}\text { ee } \\
{[\%]^{\mathrm{c}}}\end{array}$ \\
& & \\
\hline
\end{tabular}

1<smiles>c1ccc([C@@H]2CCc3ccccc3N2)cc1</smiles>

2<smiles>Cc1ccc(C2CCc3ccccc3N2)cc1</smiles>

3<smiles>Clc1ccc(C2CCc3ccccc3N2)cc1</smiles><smiles>FC(F)(F)c1ccc(C2CCc3ccccc3N2)cc1</smiles>

5<smiles>Brc1cccc([C@@H]2CCc3ccccc3N2)c1</smiles>

$6 a$

$6 b$

$6 c$

$6 d$

94

96

91

96

94

99

91

99

$6 \mathrm{e}$ more difficult to achieve. Using the optimized conditions for the fast inline reaction, we found that the continuous-flow reduction could be performed using $10 \mathrm{~mol} \%$ Brønsted acid $\mathbf{1 b}$, a flow rate of $0.1 \mathrm{~mL} \mathrm{~min}^{-1}$ and $60 \mathrm{~min}$ residence time (Table 5).

To broaden the scope of the asymmetric hydrogenations in continuous flow further, the reduction of $3 H$-indoles 9 was

Table 5: Scope of the Brønsted acid catalyzed transfer hydrogenation of quinoxalines. ${ }^{a}$

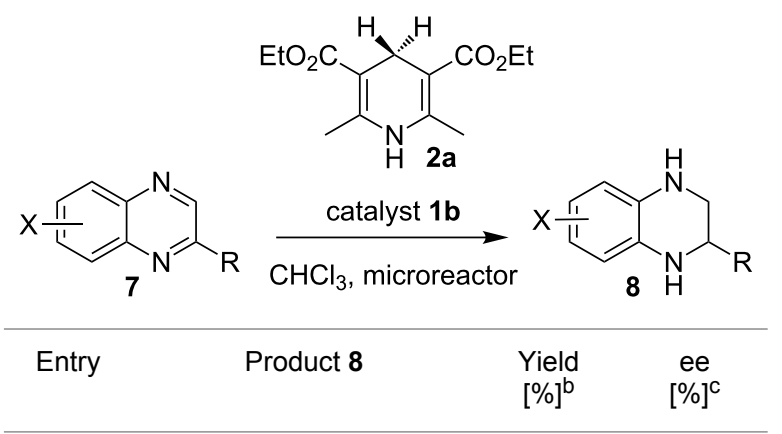

1<smiles>O=C(c1ccccc1)c1ccccc1CC1CNc2ccccc21</smiles>

77 2<smiles>Clc1ccc2c(c1)N[C@H](c1ccccc1)CN2</smiles>

68 84

3<smiles>Brc1ccc2c(c1)NC(c1ccccc1)CN2</smiles>

53 86

$8 c$<smiles>Clc1ccc(C2CNc3ccccc3N2)cc1Cl</smiles>

86

94

5<smiles>c1csc([C@@H]2CNc3ccccc3N2)c1</smiles>

$8 \mathrm{e}$
aReaction conditions: $\mathbf{5}, \mathbf{2 a}$ ( 2.4 equiv), $5 \mathrm{~mol} \% \mathbf{1 a}$ in $\mathrm{CHCl}_{3}(0.1 \mathrm{M})$ at $60{ }^{\circ} \mathrm{C}$, flow rate $0.1 \mathrm{~mL} \mathrm{~min}{ }^{-1}$, residence time $=60 \mathrm{~min}$. b/solated yields after column chromatography. ${ }^{\circ}$ Determined by chiral HPLC analysis. 
Table 6: Scope of the Brønsted acid catalyzed transfer hydrogenation of $3 \mathrm{H}$-indoles. ${ }^{a}$

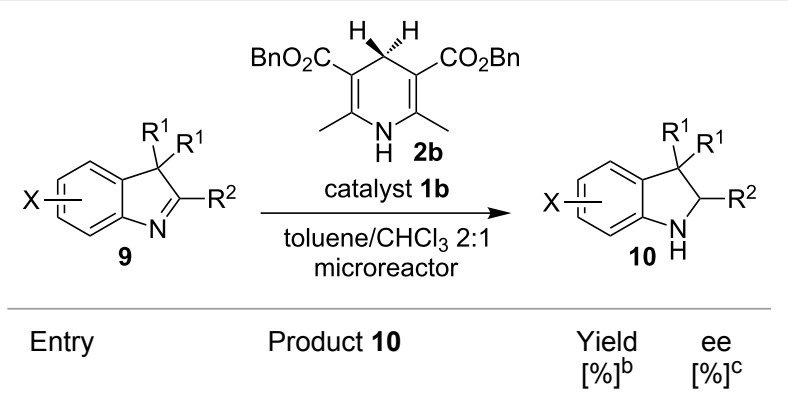

1

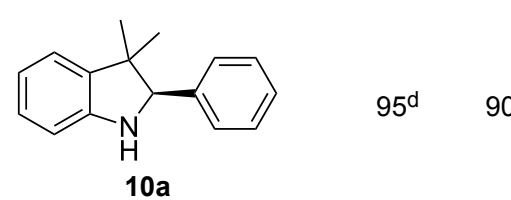

$10 a$

2

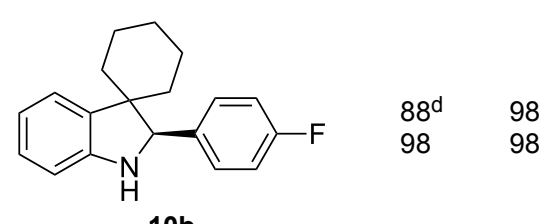

10b

3

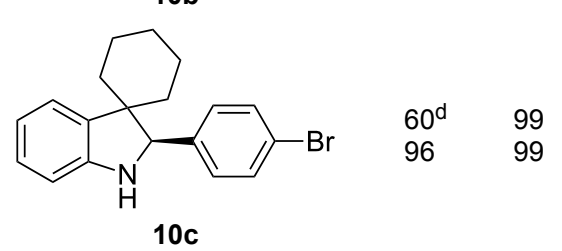

4

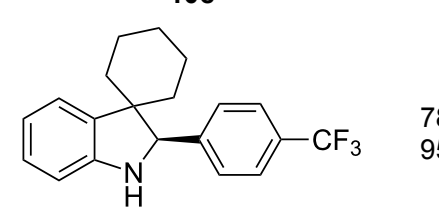

$78^{\mathrm{d}} \quad 99$

$95 \quad 99$

10d

5

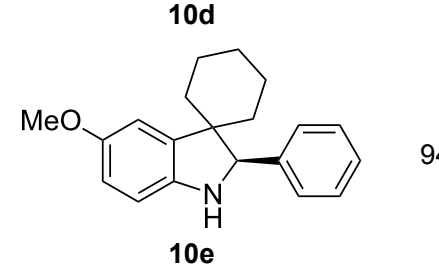

97

aReaction conditions: $\mathbf{9}, \mathbf{2 b}$ ( 1.3 equiv), $5 \mathrm{~mol} \% \mathbf{1 b}$ in toluene $/ \mathrm{CHCl}_{3}$ $(2: 1)(0.1 \mathrm{M})$ at $30{ }^{\circ} \mathrm{C}$, flow rate $0.1 \mathrm{~mL} \mathrm{~min}^{-1}$, residence time $=20 \mathrm{~min}$. ${ }^{b}$ Isolated yields after column chromatography. ${ }^{\circ}$ Determined by chiral HPLC analysis. ${ }^{d}$ Retention time: $10 \mathrm{~min}$.

studied (Table 6) [108]. Here the best reaction conditions turned out to be a temperature of $30{ }^{\circ} \mathrm{C}$, a flow rate of $0.1 \mathrm{~mL} \mathrm{~min}{ }^{-1}$, and a residence time of $20 \mathrm{~min}$. The desired indolines $\mathbf{1 0}$ were isolated in good to high yields and with excellent enantioselectivities.

\section{Conclusion}

In conclusion, we have demonstrated the potential of a microreactor setup coupled with FTIR inline analysis for monitoring asymmetric continuous-flow hydrogenations of benzoxazines, quinolines, quinoxalines and $3 H$-indoles. Following a real-time continuous-flow optimization, the corresponding products were obtained in good yields and with excellent enantioselectivities. By applying the FTIR inline monitoring, reaction parameters can be screened rapidly in a single reaction setup, and the optimal reaction conditions can be obtained much faster as compared to the classical sequence of conducting the reaction followed by analysis. Further work will include automated integration and feedback optimization of reaction parameters.

\section{Acknowledgements}

The authors acknowledge the funding by the Excellence Initiative of the German federal and state governments and the European Research Council for a starting grant.

\section{References}

1. Ehrfeld, W.; Hessel, V.; Löwe, H. Microreactors: New Technology for Modern Chemistry; Wiley-VCH: Weinheim, Germany, 2000.

2. Wirth, T., Ed. Microreactors in Organic Synthesis and Catalysis;; Wiley-VCH: Weinheim, Germany, 2008.

3. Jas, G.; Kirschning, A. Chem.-Eur. J. 2003, 9, 5708-5723. doi:10.1002/chem.200305212

4. Kikutani, Y.; Kitamori, T. Macromol. Rapid Commun. 2004, 25, 158-168. doi:10.1002/marc.200300192

5. Jähnisch, K.; Hessel, V.; Löwe, H.; Baerns, M. Angew. Chem., Int. Ed. 2004, 43, 406-446. doi:10.1002/anie.200300577

6. Doku, G. N.; Verboom, W.; Reinhoudt, D. N.; van den Berg, A. Tetrahedron 2005, 61, 2733-2742. doi:10.1016/j.tet.2005.01.028

7. Watts, P.; Haswell, S. J. Chem. Soc. Rev. 2005, 34, 235-246. doi:10.1039/b313866f

8. Geyer, K.; Codée, J. D. C.; Seeberger, P. H. Chem.-Eur. J. 2006, 12, 8434-8442. doi:10.1002/chem.200600596

9. deMello, A. J. Nature 2006, 442, 394-402. doi:10.1038/nature05062

10. Song, H.; Chen, D. L.; Ismagilov, R. F. Angew. Chem., Int. Ed. 2006, 45, 7336-7356. doi:10.1002/anie.200601554

11. Kobayashi, J.; Mori, Y.; Kobayashi, S. Chem.-Asian J. 2006, 1, 22-35. doi:10.1002/asia.200600058

12. Mason, B. P.; Price, K. E.; Steinbacher, J. L.; Bogdan, A. R.; McQuade, D. T. Chem. Rev. 2007, 107, 2300-2318. doi:10.1021/cr050944c

13. Watts, P.; Wiles, C. Chem. Commun. 2007, 443-467. doi:10.1039/b609428g

14. Ahmed-Omer, B.; Brandt, J. C.; Wirth, T. Org. Biomol. Chem. 2007, 5, 733-740. doi:10.1039/b615072a

15. Fukuyama, T.; Rahman, M. T.; Sato, M.; Ryu, I. Synlett 2008, 151-163. doi:10.1055/s-2007-1000884

16. Yoshida, J.-i.; Nagaki, A.; Yamada, T. Chem.-Eur. J. 2008, 14, 7450-7459. doi:10.1002/chem.200800582

17. Wiles, C.; Watts, P. Eur. J. Org. Chem. 2008, 1655-1671. doi:10.1002/ejoc.200701041

18. Kirschning, A. Beilstein J. Org. Chem. 2009, 5, No. 15. doi:10.3762/bjoc.5.15

19. Geyer, K.; Gustafsson, T.; Seeberger, P. H. Synlett 2009, 2382-2391. doi:10.1055/s-0029-1217828

20. Nagaki, A.; Takabayashi, N.; Tomida, Y.; Yoshida, J.-i. Beilstein J. Org. Chem. 2009, 5, No. 16. doi:10.3762/bjoc.5.16 
21. Yamada, Y. M. A.; Torii, K.; Uozumi, Y. Beilstein J. Org. Chem. 2009, 5, No. 18. doi:10.3762/bjoc.5.18

22. Brandt, J. C.; Wirth, T. Beilstein J. Org. Chem. 2009, 5, No. 30. doi:10.3762/bjoc.5.30

23. Fukuyama, T.; Rahman, M. T.; Kamata, N.; Ryu, I. Beilstein J. Org. Chem. 2009, 5, No. 34. doi:10.3762/bjoc.5.34

24. Tanaka, K.; Fukase, K. Beilstein J. Org. Chem. 2009, 5, No. 40. doi: $10.3762 /$ bjoc. 5.40

25. Kunz, U.; Turek, T. Beilstein J. Org. Chem. 2009, 5, No. 70. doi:10.3762/bjoc.5.70

26. Marre, S.; Jensen, K. F. Chem. Soc. Rev. 2010, 39, 1183-1202. doi:10.1039/b821324k

27. Yoshida, J-i.; Kim, H.; Nagaki, A. ChemSusChem 2011, 4, 331-340. doi:10.1002/cssc.201000271

28. Wegner, J.; Ceylan, S.; Kirschning, A. Chem. Commun. 2011, 47, 4583-4592. doi:10.1039/c0cc05060a

29. Min, K.-I.; Lee, T.-H.; Park, C. P.; Wu, Z.-Y.; Girault, H. H.; Ryu, I.; Fukuyama, T.; Mukai, Y.; Kim, D.-P. Angew. Chem., Int. Ed. 2010, 49, 7063-7067. doi:10.1002/anie.201002004

30. McMullen, J. P.; Stone, M. T.; Buchwald, S. L.; Jensen, K. F. Angew. Chem., Int. Ed. 2010, 49, 7076-7080. doi:10.1002/anie.201002590

31. McMullen, J. P.; Jensen, K. F. Annu. Rev. Anal. Chem. 2010, 3 19-42. doi:10.1146/annurev.anchem.111808.073718

32. Hartman, R. L.; McMullen, J. P.; Jensen, K. F. Angew. Chem., Int. Ed. 2011, 50, 7502-7519. doi:10.1002/anie.201004637

33. Shvydkiv, O.; Nolan, K.; Oelgemöller, M. Beilstein J. Org. Chem. 2011, 7, 1055-1063. doi:10.3762/bjoc.7.121

34. Nagaki, A.; Uesugi, Y.; Tomida, Y.; Yoshida, J.-i. Beilstein J. Org. Chem. 2011, 7, 1064-1069. doi:10.3762/bjoc.7.122

35. Watts, K.; Gattrell, W.; Wirth, T. Beilstein J. Org. Chem. 2011, 7, 1108-1114. doi:10.3762/bjoc.7.127

36. Roper, K. A.; Lange, H.; Polyzos, A.; Berry, M. B.; Baxendale, I. R.; Ley, S. V. Beilstein J. Org. Chem. 2011, 7, 1648-1655. doi:10.3762/bjoc.7.194

37. Saito, K.; Ueoka, K.; Matsumoto, K.; Suga, S.; Nokami, T.; Yoshida, J-i. Angew. Chem., Int. Ed. 2011, 50, 5153-5156. doi:10.1002/anie.201100854

38. Wiles, C.; Watts, P. Chem. Commun. 2011, 47, 6512-6535. doi:10.1039/c1cc00089f

39. Yoshida, J-i.; Saito, K.; Nokami, T.; Nagaki, A. Synlett 2011, 1189-1194. doi:10.1055/s-0030-1259946

40. Bogdan, A.; McQuade, D. T. Beilstein J. Org. Chem. 2009, 5, No. 17. doi:10.3762/bjoc.5.17

41. Wiles, C.; Watts, P. Green Chem. 2012, 14, 38-54. doi:10.1039/c1gc16022b

42. Wegner, J.; Ceylan, S.; Kirschning, A. Adv. Synth. Catal. 2012, 354, 17-57. doi:10.1002/adsc.201100584

43. Carter, C. F.; Baxendale, I. R.; O’Brien, M.; Pavey, J. B. J.; Ley, S. V. Org. Biomol. Chem. 2009, 7, 4594-4597. doi:10.1039/b917289k

44. Carter, C. F.; Lange, H.; Ley, S. V.; Baxendale, I. R.; Wittkamp, B.; Goode, J. G.; Gaunt, N. L. Org. Process Res. Dev. 2010, 14 , 393-404. doi:10.1021/op900305v

45. Qian, Z.; Baxendale, I. R.; Ley, S. V. Chem.-Eur. J. 2010, 16, 12342-12348. doi:10.1002/chem.201002147

46. Carter, C. F.; Baxendale, I. R.; Pavey, J. B. J.; Ley, S. V. Org. Biomol. Chem. 2010, 8, 1588-1595. doi:10.1039/b924309g

47. Leadbeater, N. E. Chem. Commun. 2010, 46, 6693-6695. doi:10.1039/c0cc01921f
48. Malet-Sanz, L.; Madrzak, J.; Ley, S. V.; Baxendale, I. R. Org. Biomol. Chem. 2010, 8, 5324-5332. doi:10.1039/c0ob00450b

49. McMullen, J. P.; Jensen, K. F. Org. Process Res. Dev. 2010, 14 , 1169-1176. doi:10.1021/op100123e

50. Foley, D. A.; Doecke, C. W.; Buser, J. Y.; Merritt, J. M.; Murphy, L.; Kissane, M.; Collins, S. G.; Maguire, A. R.; Kaerner, A. J. Org. Chem. 2011, 76, 9630-9640. doi:10.1021/jo201212p

51. Smith, C. J.; Nikbin, N.; Ley, S. V.; Lange, H.; Baxendale, I. R. Org. Biomol. Chem. 2011, 9, 1938-1947. doi:10.1039/c0ob00815j

52. Lange, H.; Carter, C. F.; Hopkin, M. D.; Burke, A.; Goode, J. G.; Baxendale, I. R.; Ley, S. V. Chem. Sci. 2011, 2, 765-769. doi:10.1039/c0sc00603c

53. Koos, P.; Gross, U.; Polyzos, A.; O’Brien, M.; Baxendale, I. R.; Ley, S. V. Org. Biomol. Chem. 2011, 9, 6903-6908. doi:10.1039/c1ob06017a

54. Keybl, J.; Jensen, K. F. Ind. Eng. Chem. Res. 2011, 50, 11013-11022. doi:10.1021/ie200936b

55. Brodmann, T.; Koos, P.; Metzger, A.; Knochel, P.; Ley, S. V. Org. Process Res. Dev. 2011. doi:10.1021/op200275d

56. Hook, B. D. A.; Dohle, W.; Hirst, P. R.; Pickworth, M.; Berry, M. B.; Booker-Milburn, K. I. J. Org. Chem. 2005, 70, 7558-7564. doi:10.1021/jo050705p

57. Wiles, C.; Watts, P.; Haswell, S. J. Tetrahedron Lett. 2007, 48, 7362-7365. doi:10.1016/j.tetlet.2007.08.027

58. Griffiths-Jones, C. M.; Hopkin, M. D.; Jönsson, D.; Ley, S. V.; Tapolczay, D. J.; Vickerstaffe, E.; Ladlow, M. J. Comb. Chem. 2007, 9, 422-430. doi:10.1021/cc060152b

59. Mennecke, K.; Solodenko, W.; Kirschning, A. Synthesis 2008, 1589-1599. doi:10.1055/s-2008-1072579

60. Mennecke, K.; Kirschning, A. Synthesis 2008, 3267-3272. doi:10.1055/s-2008-1067274

61. Baxendale, I. R.; Ley, S. V.; Mansfield, A. C.; Smith, C. D. Angew. Chem., Int. Ed. 2009, 48, 4017-4021. doi:10.1002/anie.200900970

62. Wang, N.; Matsumoto, T.; Ueno, M.; Miyamura, H.; Kobayashi, S. Angew. Chem., Int. Ed. 2009, 48, 4744-4746. doi:10.1002/anie.200900565

63. Brasholz, M.; Macdonald, J. M.; Saubern, S.; Ryan, J. H.; Holmes, A. B. Chem.-Eur. J. 2010, 16, 11471-11480. doi:10.1002/chem.201001435

64. Costantini, F.; Benetti, E. M.; Tiggelaar, R. M.; Gardeniers, H. J. G. E.; Reinhoudt, D. N.; Huskens, J.; Vancso, G. J.; Verboom, W. Chem.-Eur. J. 2010, 16, 12406-12411. doi:10.1002/chem.201000948

65. Gutmann, B.; Roduit, J.-P.; Roberge, D.; Kappe, C. O. Angew. Chem., Int. Ed. 2010, 49, 7101-7105. doi:10.1002/anie.201003733

66. Wahab, B.; Ellames, G.; Passey, S.; Watts, P. Tetrahedron 2010, 66, 3861-3865. doi:10.1016/j.tet.2010.03.005

67. Fuse, S.; Tanabe, N.; Yoshida, M.; Yoshida, H.; Doi, T.; Takahashi, T. Chem. Commun. 2010, 46, 8722-8724. doi:10.1039/c0cc02239j

68. Venturoni, F.; Nikbin, N.; Ley, S. V.; Baxendale, I. R. Org. Biomol. Chem. 2010, 8, 1798-1806. doi:10.1039/b925327k

69. Webb, D.; Jamison, T. F. Chem. Sci. 2010, 1, 675-680. doi:10.1039/c0sc00381f

70. Gutmann, B.; Roduit, J.-P.; Roberge, D.; Kappe, C. O. Chem.-Eur. J. 2011, 17, 13146-13150. doi:10.1002/chem.201102772

71. Maurya, R. A.; Park, C. P.; Lee, J. H.; Kim, D.-P. Angew. Chem., Int. Ed. 2011, 50, 5952-5955. doi:10.1002/anie.201101977 
72. Sniady, A.; Bedore, M. W.; Jamison, T. F. Angew. Chem., Int. Ed. 2011, 50, 2155-2158. doi:10.1002/anie.201006440

73. Li, P.; Buchwald, S. L. Angew. Chem., Int. Ed. 2011, 50, 6396-6400. doi:10.1002/anie.201102401

74. Noël, T.; Maimone, T. J.; Buchwald, S. L. Angew. Chem., Int. Ed. 2011, 50, 8900-8903. doi:10.1002/anie.201104652

75. Shu, W.; Pellegatti, L.; Oberli, M. A.; Buchwald, S. L. Angew. Chem., Int. Ed. 2011, 50, 10665-10669. doi:10.1002/anie.201105223

76. O'Brien, A. G.; Lévesque, F.; Seeberger, P. H. Chem. Commun. 2011, 47, 2688-2690. doi:10.1039/c0cc04481d

77. Noël, T.; Buchwald, S. L. Chem. Soc. Rev. 2011, 40, 5010-5029. doi:10.1039/c1cs15075h

78. Kim, H.; Nagaki, A.; Yoshida, J.-i. Nat. Commun. 2011, 264 doi:10.1038/ncomms1264

79. Browne, D. L.; Baumann, M.; Harji, B. H.; Baxendale, I. R.; Ley, S. V. Org. Lett. 2011, 13, 3312-3315. doi:10.1021/ol2010006

80. Allian, A. D.; Richter, S. M.; Kallemeyn, J. M.; Robbins, T. A.; Kishore, V. Org. Process Res. Dev. 2011, 15, 91-97. doi:10.1021/op100249z

81. Wiles, C.; Wattts, P.; Haswell, S. J.; Pombo-Villar, E. Lab Chip 2004, 4, 171-173. doi:10.1039/b400280f

82. Jönsson, C.; Lundgren, S.; Haswell, S. J.; Moberg, C. Tetrahedron 2004, 60, 10515-10520. doi:10.1016/j.tet.2004.08.080

83. de Bellefon, C.; Lamouille, T.; Pestre, N.; Bornette, F.; Pennemann, H.; Neumann, F.; Hessel, V. Catal. Today 2005, 110 , 179-187. doi:10.1016/j.cattod.2005.09.002

84. Hamberg, A.; Lundgren, S.; Wingstrand, E.; Moberg, C.; Hult, K. Chem.-Eur. J. 2007, 13, 4334-4341. doi:10.1002/chem.200601638

85. Sakeda, K.; Wakabayashi, K.; Matsushita, Y.; Ichimura, T.; Suzuki, T.; Wada, T.; Inoue, Y. J. Photochem. Photobiol., A 2007, 192, 166-171. doi:10.1016/j.jphotochem.2007.05.019

86. Mak, X. Y.; Laurino, P.; Seeberger, P. H. Beilstein J. Org. Chem. 2009, 5, No. 19. doi:10.3762/bjoc.5.19

87. Shi, L.; Wang, X.; Sandoval, C. A.; Wang, Z.; Li, H.; Wu, J.; Yu, L.; Ding, K. Chem.-Eur. J. 2009, 15, 9855-9867. doi:10.1002/chem.200900899

88. Rolland, J.; Cambeiro, X. C.; Rodríguez-Escrich, C.; Pericàs, M. A. Beilstein J. Org. Chem. 2009, 5, No. 56. doi:10.3762/bjoc.5.56

89. Alza, E.; Rodríguez-Escrich, C.; Sayalero, S.; Bastero, A.; Pericàs, M. A. Chem.-Eur. J. 2009, 15, 10167-10172. doi:10.1002/chem.200901310

90. Tomida, Y.; Nagaki, A.; Yoshida, J.-i. J. Am. Chem. Soc. 2011, 133, 3744-3747. doi:10.1021/ja110898s

91. Carter, C. F.; Lange, H.; Sakai, D.; Baxendale, I. R.; Ley, S. V. Chem.-Eur. J. 2011, 17, 3398-3405. doi:10.1002/chem.201003148

92. Massi, A.; Cavazzzini, A.; Del Zoppo, L.; Pandoli, O.; Costa, V.; Pasti, L.; Giovannini, P. P. Tetrahedron Lett. 2011, 52, 619-622. doi:10.1016/j.tetlet.2010.11.157

93. Takeda, K.; Oohara, T.; Shimada, N.; Nambu, H.; Hashimoto, S. Chem.-Eur. J. 2011, 17, 13992-13998. doi:10.1002/chem.201102733

94. Fritzsche, S.; Ohla, S.; Glaser, P.; Giera, D. S.; Sickert, M.; Schneider, C.; Belder, D. Angew. Chem., Int. Ed. 2011, 50, 9467-9470. doi:10.1002/anie.201102331

95. Cambeiro, X. C.; Martín-Rapún, R.; Miranda, P. O.; Sayalero, S.; Alza, E.; Llanes, P.; Pericàs, M. A. Beilstein J. Org. Chem. 2011, 7, 1486-1493. doi:10.3762/bjoc.7.172

96. Ayats, C.; Henseler, A. H.; Pericàs, M. A. ChemSusChem 2012, 5, 320-325. doi:10.1002/cssc.201100570
97. Rueping, M.; Sugiono, E.; Azap, C.; Theissmann, T.; Bolte, M. Org. Lett. 2005, 7, 3781-3783. doi:10.1021/ol0515964

98. Rueping, M.; Antonchick, A. P. Angew. Chem., Int. Ed. 2007, 46, 4562-4565. doi:10.1002/anie.200701158

99. Rueping, M.; Sugiono, E.; Schoepke, F. R. Synlett 2010, 852-865. doi:10.1055/s-0029-1219528

100.Rueping, M.; Merino, E.; Koenigs, R. M. Adv. Synth. Catal. 2010, 352, 2629-2634. doi:10.1002/adsc.201000547

101.Rueping, M.; Dufour, J.; Schoepke, F. R. Green Chem. 2011, 13, 1084-1105. doi:10.1039/c1gc15027h

102. Rueping, M.; Antonchick, A. P.; Theissmann, T. Angew. Chem., Int. Ed. 2006, 45, 6751-6755. doi:10.1002/anie.200601832

103. Rueping, M.; Antonchick, A. P.; Theissmann, T. Angew. Chem., Int. Ed. 2006, 45, 3683-3686. doi:10.1002/anie.200600191

104.Rueping, M.; Theissmann, T.; Raja, S.; Bats, J. W. Adv. Synth. Catal. 2008, 350, 1001-1006. doi:10.1002/adsc.200800020

105.Rueping, M.; Stoeckel, M.; Sugiono, E.; Theissmann, T. Tetrahedron 2010, 66, 6565-6568. doi:10.1016/j.tet.2010.04.091

106. Rueping, M.; Theissmann, T.; Stoeckel, M.; Antonchick, A. P. Org. Biomol. Chem. 2011, 9, 6844-6850. doi:10.1039/c1ob05870c

107.Rueping, M.; Tato, F.; Schoepke, F. R. Chem.-Eur. J. 2010, 16, 2688-2691. doi:10.1002/chem.200902907

108. Rueping, M.; Brinkmann, C.; Antonchick, A. P.; Atodiresei, I. Org. Lett. 2010, 12, 4604-4607. doi:10.1021/ol1019234

\section{License and Terms}

This is an Open Access article under the terms of the Creative Commons Attribution License (http://creativecommons.org/licenses/by/2.0), which permits unrestricted use, distribution, and reproduction in any medium, provided the original work is properly cited.

The license is subject to the Beilstein Journal of Organic Chemistry terms and conditions:

(http://www.beilstein-journals.org/bjoc)

The definitive version of this article is the electronic one which can be found at: doi:10.3762/bjoc. 8.32 